\title{
Macroprolactinoma Resistente aos Agonistas Dopaminérgicos: Relato de Caso
}

\author{
Macroprolactinoma Resistant to Dopamine Agonists: a Case Report \\ Rosana Maria dos Reis ${ }^{1}$, Camila Toffoli Ribeiro ${ }^{1}$, Marcos Felipe de Silva Sá ${ }^{1}$, \\ Hélio Humberto Angotti Carrara ${ }^{1}$, Hélio Rubens Machado ${ }^{2}$, Antônio Carlos dos Santos ${ }^{3}$
}

\begin{abstract}
RESUM0
Os macroprolactinomas são tumores benignos hipofisários, secretores de prolactina, manifestando-se com amenorréia, galactorréia e disfunção gonadal. O tratamento de primeira escolha é clínico, com agonistas dopaminérgicos. O tratamento cirúrgico é reservado para os raros casos que não respondem ao tratamento clínico, intolerância à medicação ou nos casos agudos quando ocorre a hemorragia intra-tumoral. Descrevemos o caso de uma paciente portadora de macroprolactinoma submetida a duas ressecções cirúrgicas e resistente ao tratamento clínico, com evolução não usual.
\end{abstract}

PALAVRAS-CHAVE: Prolactinomas. Bromocriptina. Cabergolina. Cirurgia transesfenoidal. Hiperprolactinemia

\section{Introdução}

A sindrome hiperprolactinêmica é doença freqüente, com incidência de 1 a $10 \%$ na população geral ${ }^{1}$. Manifesta-se clinicamente como disfunção gonadal (ciclos oligomenorréicos ou amenorréia), galactorréia, diminuição da libido, infertilidade e mais raramente sintomas devidos a compressão tumoral, como alterações do campo visual e cefaléia.

Uma das causas mais freqüentes de hiperprolactinemia são os tumores hipofisários secretores de prolactina (PRL), que elevam seus níveis plasmáticos geralmente a valores superiores a $50 \mathrm{ng} / \mathrm{mL}$. Assim, sempre que houver sus-

${ }^{1}$ Setor de Ginecologia Endócrina e Reprodução Humana do Departamento de Ginecologia e Obstetrícia

${ }^{2}$ Setor de Neurocirurgia do Departamento de Cirurgia e Anatomia

${ }^{3}$ Setor de Neurorradiologia do Departamento de Cirurgia e Anatomia

Faculdade de Medicina de Ribeirão Preto da Universidade de São Paulo

Correspondência:

Rosana Maria Reis

Departamento de Ginecologia e Obstetrícia

Av. dos Bandeirantes, $\mathrm{n}^{\circ} .3900$

14049-900 - Ribeirão Preto - SP

Fone: (16) 602-2926

Fax: (16) 633-0946

e-mail: romareis@fmrp.usp.br peita clínica dessas neoplasias ou níveis de PRL acima dos citados deve ser feito estudo do encéfalo e sela túrcica por exames de imagem².

A imagem de ressonância magnética (IRM) é o exame de escolha para avaliação da sela túrcica, devido à maior sensibilidade para detecção de pequenos tumores e maior acurácia na avaliação da extensão de prolactinomas de maiores dimensões ${ }^{3}$. Estas neoplasias são classificadas de acordo com o diâmetro de seu maior eixo em microprolactinomas (menor que $10 \mathrm{~mm}$ ) ou macroprolatinomas (maior ou igual a $10 \mathrm{~mm}$ ).

O tratamento clínico é atualmente considerado a primeira opção na abordagem de todas as causas de hiperprolactinemia, incluindo as tumorais. Descrevem-se taxas de sucesso, com obtenção de normoprolactinemia, de até $90 \%$, com redução de $60 \%$ do volume tumoral em média ${ }^{4}$.

A droga mais antiga e classicamente utilizada para tal fim é a bromocriptina, alcalóide derivado do ergot, que se liga inespecificamente a receptores dopaminérgicos D1 e D2, apresenta meia-vida de 8 a 12 horas e atua inibindo a sintese e secreção de PRL pelos lactotrofos ${ }^{5}$. Como alternativa mais recente, surgiu a cabergolina, agonista dopaminérgico com meia-vida mais longa, de ação específica sobre os receptores D2 e com menos efeitos adversos ${ }^{6}$. 
Tem despertado crescente interesse a comparação da eficácia entre as duas drogas, e estudos recentes têm apontado a superioridade da cabergolina no que se refere à obtenção de níveis séricos normais de PRL, com restauração da função gonadal, desaparecimento da galactorréia, recuperação da capacidade reprodutiva e redução do volume tumoral de micro e macroprolactinomas ${ }^{7-9}$.

O tratamento cirúrgico dos prolactinomas deve ser reservado para os casos em que não há resposta ao tratamento clínico, quando o paciente não tolera os efeitos adversos das medicações ou na vigência de hemorragia intratumoral, situação em que a cirurgia constitui tratamento de emergência. Há controvérsias sobre a necessidade de cirurgia na presença de sintomas visuais, conseqüentes ao efeito da massa tumoral por compressão do quiasma óptico. Enquanto alguns autores indicam procedimento cirúrgico rotineiramente em tais circunstâncias ${ }^{5}$, outros defendem tentativa inicial de tratamento clínico, pois se observou melhora rápida dos sintomas, até mesmo nas primeiras 24 horas que sucedem à administração do medicamento ${ }^{10}$. Finalmente deve-se lembrar que o tratamento clínico pode complementar o tratamento cirúrgico em casos nos quais não houve remoção total do adenoma e os niveis de PRL permaneceram ainda elevados depois da cirurgia. O relato de caso a seguir ilustra evolução insatisfatória de uma paciente com macroprolactinoma, submetida a tratamento cirúrgico e clínico, com resistência aos agonistas dopaminérgicos.

\section{Relato de Caso}

M.L.V.S., branca, 25 anos, nuligesta, procedente de Bebedouro - SP, foi atendida no serviço de Ginecologia Endócrina do Hospital das Clínicas da Faculdade de Medicina - USP Ribeirão Preto em março de 1996, referindo ciclos menstruais irregulares desde a menarca. Encontrava-se em amenorréia por quatro anos aproximadamente e com galactorréia desde o início do quadro. Havia iniciado investigação com facultativo em sua cidade, tendo sido solicitadas dosagens de PRL (níveis superiores a $1500 \mathrm{ng} / \mathrm{mL}$ ), radiografia de sela túrcica e tomografia computadorizada (TC), com diagnóstico de macroprolactinoma.

Foi iniciado tratamento com bromocriptina, atingindo-se a dose de $25 \mathrm{mg} / \mathrm{dia}$, com diminuição dos níveis da PRL para $160 \mathrm{ng} / \mathrm{mL}$, porém com aumento progressivo posteriormente. Foi submetida a adenomectomia em fevereiro de 1995, apresentando níveis de PRL de $160 \mathrm{ng} / \mathrm{mL}$ no pós-operatório. Após período de quatro meses, os níveis de
PRL voltaram a elevar-se, atingindo $1600 \mathrm{ng} / \mathrm{mL}$. Foi reiniciada bromocriptina, em uso de $40 \mathrm{mg} /$ dia, e nesse momento a paciente passou a ser acompanhada em nosso Serviço (Figura 1).
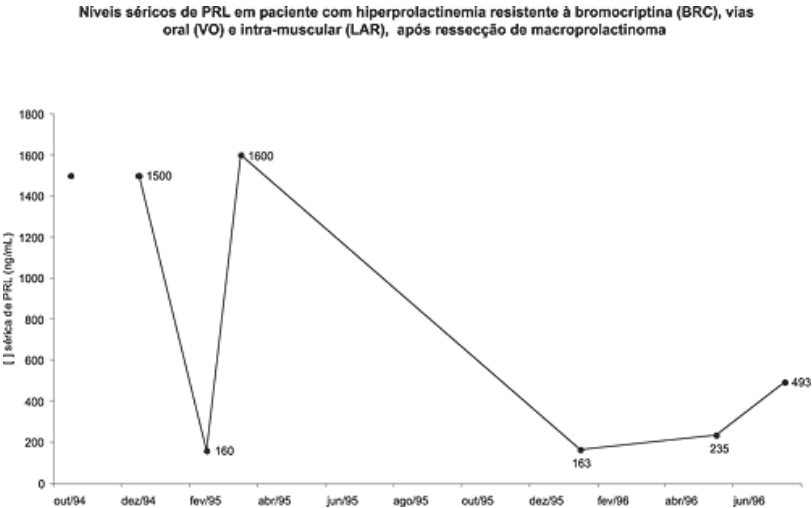

Figura 1 - Níveis séricos de prolactina (PRL) em $\mathrm{ng} / \mathrm{mL}$ antes e após o tratamento cirúrgico e com uso de doses crescentes de bromocriptina por via oral (BRC VO) e em forma de depósito (LAR).

A paciente trazia TC recente, com o diagnóstico de hipófise com dimensões no limite superior da normalidade, e dosagem de PRL com valor de $163 \mathrm{ng} / \mathrm{mL}$.

Em abril de 1996 optou-se por iniciar bromocriptina de depósito (LAR) a $50 \mathrm{mg} / \mathrm{mês}$. Porém, os níveis de PRL permaneceram elevados, sendo de 493,0 $\mathrm{ng} / \mathrm{mL}, 16$ dias após o uso da medicação. Foi aumentada a dose para uma ampola de 15 em 15 dias.

No entanto, a paciente perdeu seguimento, ficando afastada do Serviço durante um ano e 8 meses. Referia ter feito uso da medicação prescrita apenas durante 3 meses. Quando retomou seguimento no Serviço, a TC demonstrava processo expansivo selar com expansão para-selar, com os niveis de PRL atingindo $9061 \mathrm{ng} / \mathrm{mL}$. A paciente queixava-se de cefaléia persistente e galactorréia à expressão.

O quadro clínico agravou-se e antes da realização de novos exames, apresentou quadro de cefaléia de forte intensidade, vômitos, paralisia facial à esquerda, ptose palpebral e evolução para coma. Foi atendida no mesmo Serviço em que fora realizada a primeira cirurgia, com diagnóstico de hemorragia intratumoral (apoplexia hipofisária) (Figura 2), sendo realizado novo procedimento de ressecção transesfenoidal de urgência em $25 /$ março/98. Cinco dias após a cirurgia foi feita IRM controle de e dosagem de PRL sérica, que foi de $4135 \mathrm{ng} / \mathrm{mL}$. A paciente foi dispensada em uso de dexametasona (12 $\mathrm{mg} /$ dia) e bromocriptina a $10 \mathrm{mg} /$ dia.

A dose de bromocriptina foi aumentada gradativamente, chegando-se a $30 \mathrm{mg} /$ dia em janeiro de 1999. A IRM mostrava melhora do padrão 
de imagem, com diminuição da massa tumoral e estabilização em exames subseqüentes (Figura 2). No entanto, a PRL manteve-se elevada durante todo o tratamento, variando de $284 \mathrm{ng} / \mathrm{mL}$ a 3483 $\mathrm{ng} / \mathrm{mL}$ (Figura 3).
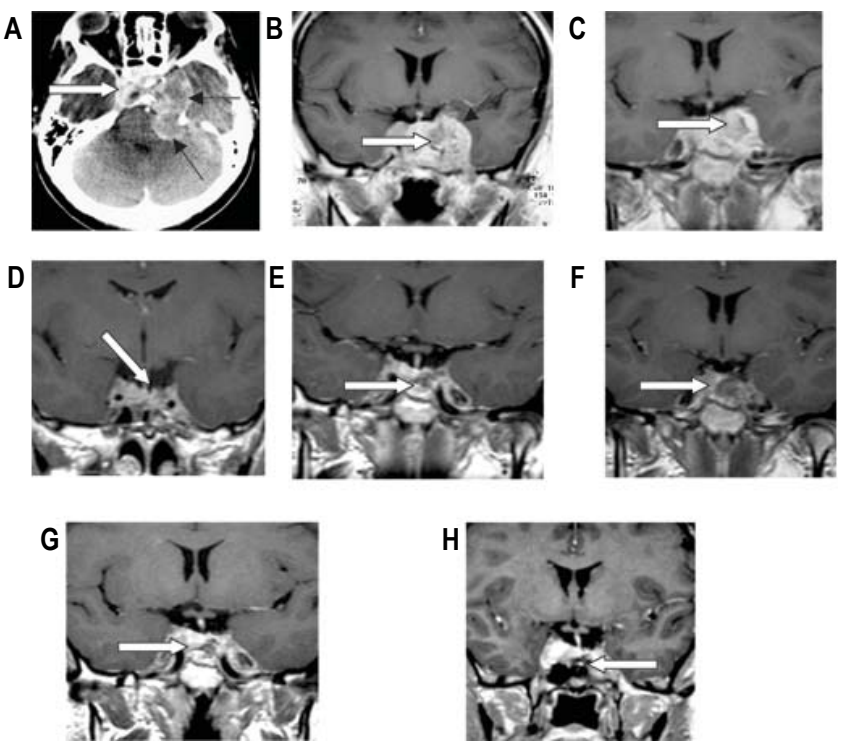

A - (25/3/98) Lesão hiperdensa comprimindo o tronco (seta branca), representada po apoplexia tumoral (setas pretas). Paciente em coma. B - (30/3/98) - Paciente no $5^{\circ}$. pósoperatório de adenomectomia transesfenoidal de urgência, com o macroadenoma mostrando sangramento em seu interior, com invasão do seio cavernoso. C - (17/6/98) - Persistência da lesão com hemorragia paraselar e extensão para região infratentorial. D - (08/6/99) e E - (24/4/00) - Sinais de invasão de seio cavernoso à direita. F - (5/1/ 01), G - (7/2/03) e H - (4/3/04) - Manutenção do padrão aos exames de imagem, apesar de niveis de prolactina crescentes.

Figura 2 - Seguimento do macroadenoma por exames de imagem.

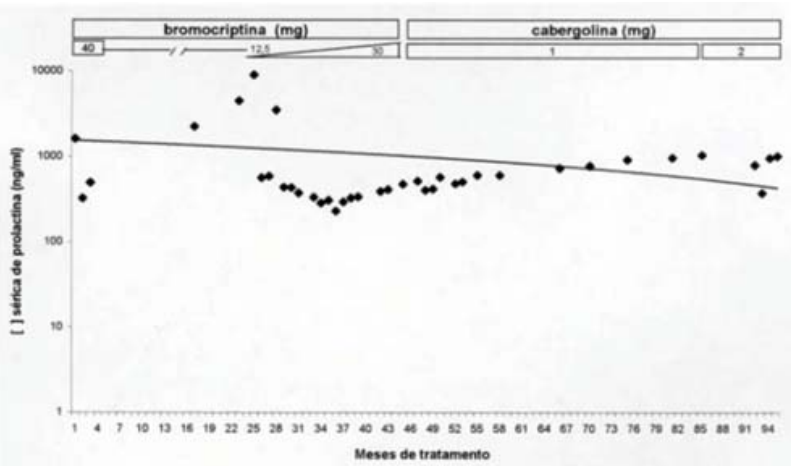

Figura 3 - Níveis séricos de prolactina $(\mathrm{ng} / \mathrm{mL})$ sob administração das duas drogas dopaminérgicas. Observar escala logarítmica dos valores de prolactina.

Realizou-se pesquisa de formas biologicamente menos ativas de PRL (big ou big-big), aventando-se a hipótese de secreção aberrante de formas moleculares poliméricas, o que poderia explicar niveis tão elevados de PRL a despeito de tamanho tumoral estável. Contudo, a cromatografia demonstrou tratar-se predominantemente da forma little, de $22 \mathrm{kDa}$ (Figura 4).

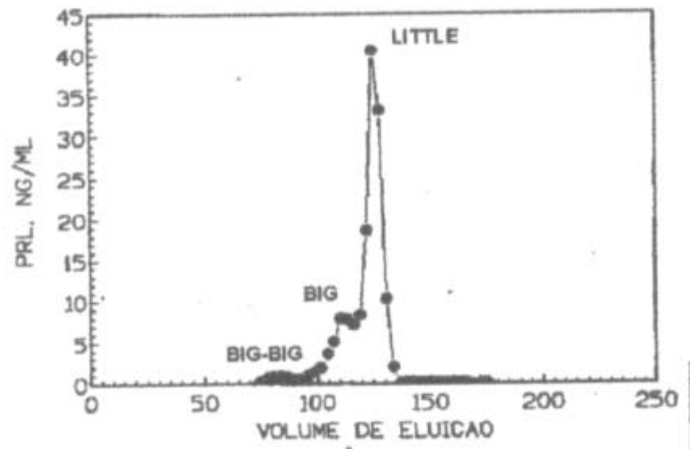

Figura 4 - Estudo cromatográfico das formas moleculares de prolactina (eluição em Sephacryl S-200), demonstrando haver predominância da forma little.

Em dezembro de 1999, como a paciente mantinha niveis elevados de PRL (maiores que $400 \mathrm{ng}$ / $\mathrm{mL}$ ), optou-se por substituir a bromocriptina pela cabergolina, com dose inicial de $1 \mathrm{mg} /$ semana, chegando-se até $2 \mathrm{mg} / \mathrm{semana}$, sem qualquer diminuição da prolactinemia.

A paciente mantém-se em amenorréia há mais de dez anos, e a PRL voltou a atingir valores superiores a $1000 \mathrm{ng} / \mathrm{mL}$ em março de 2003. Clinicamente apresenta alopecia, obesidade e anisocoria, além do desejo de concepção, choro fácil e anedonia. Atualmente a dose da cabergolina foi aumentada para $4 \mathrm{mg} / \mathrm{semana}$, porém os níveis de PRL mantêm-se em $880 \mathrm{ng} / \mathrm{mL}$.

Os exames de função hipofisária mantiveram-se normais ao longo do seguimento, não tendo sido notados sinais clínicos ou laboratoriais de hipopituitarismo. A função gonadal não foi restabelecida, o que resultou em atrofia nos esfregaços cérvico-vaginais. O hipoestrogenismo foi laboratorialmente confirmado, com dosagens de estradiol inferiores a $20 \mathrm{pg} / \mathrm{mL}$. A avaliação da massa óssea foi feita por meio de densitometria, com achado de osteopenia no primeiro exame, que no entanto normalizou-se dois anos após, apenas com orientações higieno-dietéticas e suplementação de carbonato de cálcio. A campimetria manteve-se normal durante o tratamento.

\section{Discussão}

Este caso ilustra múltiplos aspectos de interesse sobre a evolução de macroprolactinomas. A situação descrita não foi, absolutamente, a esperada do habitual destas neoplasias benignas da hipófise. A maioria dos prolactinomas, que constituem 40\% dos tumores hipofisários, respondem 
satisfatoriamente, independentemente da abordagem empregada ${ }^{11}$.

O tratamento cirúrgico apresenta elevados indices de recidiva, de 26 a 50\% ${ }^{10}$, mesmo quando realizado por cirurgiões experientes de grandes centros. Nas duas ocasiões em que a paciente foi submetida a adenomectomia tinha indicações precisas para tal, por resistência ao tratamento clínico quando indicada a primeira cirurgia e por hemorragia intratumoral no segundo procedimento, constituindo tratamento emergencial. Relata-se na literatura que os niveis de PRL no pós-operatório precoce são um dos principais indicadores da eficiência do tratamento cirúrgico. Os niveis de PRL no primeiro dia de pós-operatório inferiores a 10 $\mathrm{ng} / \mathrm{mL}$ para alguns autore ${ }^{12}$ ou a $5 \mathrm{ng} / \mathrm{mL}$ para outros ${ }^{5}$ são o fator que melhor se correlaciona com a cura. No caso relatado, portanto, a paciente apresentava chances escassas de boa evolução somente com estes parâmetros, principalmente após a segunda cirurgia, quando os níveis de PRL foram de $4135 \mathrm{ng} / \mathrm{mL}$ no quinto dia pós-operatório.

Outro conceito ilustrado foi o de resistência ao tratamento com agonistas dopaminérgicos. A resistência à bromocriptina é definida como ausência de normalização dos níveis de PRL e/ou redução do volume tumoral após pelo menos três meses de tratamento com $15 \mathrm{mg} / \mathrm{dia}^{13}$. A causa deste fenômeno não é bem compreendida, porém aventa-se que possa haver ausência ou pouca expressão de receptores D2 na superficie da membrana celular das células tumorais, ou que um defeito pós-receptor esteja implicado ${ }^{14}$.

Por outro lado, a cabergolina parece ter efeito favorável em pacientes que não respondem à bromocriptina. Colao et al. ${ }^{8}$, em estudo de 19 casos de macroprolactinomas resistentes à bromocriptina, obtiveram normalização da PRL em 9 e redução significativa em 9 dos demais. Apenas um paciente permaneceu sem resposta alguma à medicação. Ainda neste estudo foi detectada diminuição significativa do tamanho tumoral em 9 dos pacientes estudados.

Em estudo no qual se compararam as duas drogas, Di Sarno et al. ${ }^{9}$ observaram que a cabergolina é superior à bromocriptina em relação à eficácia para promover normoprolactinemia em pacientes com macroprolactinomas $(82,1$ vs $46,4 \%$ ). Achado intrigante neste estudo foi o fato de a cabergolina ter sido capaz de causar redução do volume tumoral em todos os pacientes tratados, mesmo quando não houve normalização nos niveis de PRL, ao passo que com o uso da bromocriptina, esse efeito não foi constatado.

No presente caso houve resistência às duas drogas, e a cabergolina não provocou o efeito de redução das dimensões tumorais. Houve, contu- do, manutenção das dimensões da neoplasia. O resultado encontrado poderia ser explicado pela hipótese de que em macroprolactinomas gigantes os niveis séricos de PRL não têm boa correlação com o volume tumoral observado em exames de imagem, conforme apontado por Shrivastava et al. ${ }^{10}$, ao verificar este mesmo achado em série de dez pacientes com este raro subgrupo de neoplasias hipofisárias. Assim, o fato de a PRL ter se mantido em níveis elevados não implica necessariamente falha de tratamento.

É ainda questão controversa a validade da administração de doses elevadas de cabergolina em pacientes resistentes a esta droga. Vários trabalhos descrevem uso de doses de 0,5 a $3 \mathrm{mg} / \mathrm{se}-$ mana ${ }^{8,15}$, embora a maioria dos tumores responda a doses de $2 \mathrm{mg} / \mathrm{semana}$. Apesar de recentemente terem surgido relatos de casos isolados com uso de doses muito altas de cabergolina (até 21 $\mathrm{mg} / \mathrm{semana}$, com as quais se obtiveram normoprolactinemia e restauração da função gonadal ${ }^{7}$, alguns estudiosos do assunto afirmam não haver beneficio adicional com o uso de doses superiores a $3 \mathrm{mg} /$ semana $^{9}$.

Em relação ao estudo cromatográfico das formas moleculares de PRL, o resultado encontrado foi compatível com a apresentação clínica da paciente. Seria surpreendente a detecção de predominância de formas poliméricas da molécula, em detrimento da forma small, uma vez que nas macroprolactinemias o quadro clínico costuma ser oligossintomático ${ }^{16}$, enquanto a paciente em questão apresentava amenorréia prolongada e galactorréia. Tem sido encontrada baixa ocorrência de macroprolactinemia entre pacientes com macroprolactinomas. Em pacientes com macroprolactinomas os exames de imagem são normais em $78,9 \%$ das vezes, e nenhum dos exames alterados deveu-se a macroadenomas ${ }^{17}$. Mounier et al. ${ }^{16}$, ao estudarem pacientes com hiperprolactinemia de etiologia tumoral, encontraram predominância das formas poliméricas em $38 \%$. No entanto, em todas tratavam-se de microprolactinomas.

Outra modalidade terapêutica que poderia ter sido associada no presente caso seria a radioterapia. No entanto, esta vem sendo empregada primariamente em casos de evolução progressiva tumoral ou comprometimento de nervos cranianos. A radioterapia convencional pode estar associada a indices de lesão do parênquima induzida por radiação de até $30 \%^{18}$, deixando seqüelas permanentes. A radiocirurgia por estereotaxia (gamma knife) é apontada como alternativa atraente, com menor taxa de lesões por radiação ${ }^{19}$ e controle eficaz do tamanho tumoral ${ }^{19,20}$. Todavia, os efeitos a longo prazo e a dose segura ainda não estão bem definidos na literatura, e aponta-se que o índice de comprome- 
timento da função hipofisária pode ser significativo, especialmente em tumores de volume maior, quando são necessárias doses mais elevadas de radiação, e em pacientes previamente submetidos a tratamento cirúrgico ${ }^{21}$. Como no caso estudado a paciente apresenta estabilidade da lesão em IRM seriadas, e não há comprometimento significativo de nervos cranianos (exceto por anisocoria), a radioterapia não foi considerada boa alternativa e optouse por reservá-la para o futuro caso haja nova progressão das dimensões tumorais.

Apesar de atualmente obterem-se altas taxas de sucesso quer com o tratamento clinico, quer com o cirúrgico associado, devemos ter em mente que o tratamento dos prolactinomas deve ser individualizado e o caso em apreço ilustra muito bem que alguns pacientes ainda representam um desafio à terapêutica vigente.

\section{ABSTRACT}

Macroprolactinomas are benign prolactin-secreting pituitary tumors, causing amenorrhea, galactorrhea and gonadal dysfunction. Clinical treatment with dopamine agonists is the first-choice therapy. Surgery is indicated for the rare cases that are resistant to clinical treatment, when there is intolerance to the medication, or intratumoral hemorrhage is detected. We describe the case of a female patient with macroprolactinoma submitted to two surgical procedures and resistant to clinical treatment, with unusual evolution.

KEYWORDS: Prolactinomas. Bromocriptine. Cabergoline. Transsphenoidal surgery. Hyperprolactinemia.

\section{Referências}

1. Molitch ME. Prolactin. In: Melmed S, editor. The Pituitary. $1^{\text {st }}$ ed. Cambridge: Blackwell Science; 1995. p. 136-86.

2. Silva de Sá MF. Síndrome hiperprolactinêmica: galactorréia. In: Halbe HW, editor. Tratado de

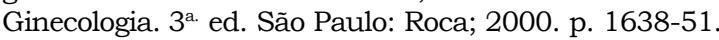

3. Naidich MJ, Russell EJ. Current approaches to imaging of the sellar region and pituitary. Endocrinol Metab Clin North Am 1999; 28:45-79.

4. Colao A, Merola B, Sarnacchiaro F, et al. Comparison among different dopamine-agonists of new formulation in the clinical management of macroprolactinomas. Horm Res 1995; 44:222-8.

5. Tella Júnior OI, Herculano MA, Delcello R, Aguiar PH. Prolactinomas: aspectos neurocirúrgicos. Arq Neuropsiquiatr 2002; 60:106-12.

6. Ferrari C, Barbieri C, Caldara R, et al. Long-lasting prolactin-lowering effect of cabergoline, a new dopamine agonist, in hyperprolactinemic patients. J Clin Endocrinol Metab 1986; 63:941-5.

7. Gillam MP, Middler S, Freed DJ, Molitch ME. The novel use of very high doses of cabergoline and a combination of testosterone and an aromatase inhibitor in the treatment of a giant prolactinomas. J Clin Endocrinol Metab 2002; 87:4447-51.

8. Colao A, Di Sarno A, Sarnacchiaro F, et al. Prolactinomas resistant to standard dopamine agonists respond to chronic cabergoline treatment. J Clin Endocrinol Metab 1997; 82:876-83.

9. Di Sarno A, Landi ML, Cappabianca P, et al. Resistance to cabergoline as compared with bromocriptine in hyperprolactinemia: prevalence, clinical definition, and therapeutic strategy. J Clin Endocrinol Metab 2001; 86:5256-61.

10.Shrivastava RK, Arginteanu MS, King WA, Post KD. Giant prolactinomas: clinical management and longterm follow up. J Neurosurg 2002; 97:299-306.

11. Ciric I. Long-term management and outcome for pituitary tumors. Neurosurg Clin N Am 2003; 14:167-71.

12.Amar AP, Couldwell WT, Chen JC, Weiss MH. Predictive value of serum prolactin levels measured immediately after transsphenoidal surgery. J Neurosurg 2002; 97:307-14.

13.Kovacs K, Stefaneanu L, Hovarth E, Buchfelder M, Fahlbusch R, Becker W. Prolactin-producing pituitary tumor: resistance to dopamine agonist therapy. Case Report. J Neurosurg 1995; 82:886-90.

14. Brue T, Pellegrini I, Priou A, Morange I, Jaquet P. Prolactinomas and resistance to dopamine agonists. Horm Res 1992; 38:84-9.

15. Colao A, Di Sarno A, Landi ML, et al. Long-term and low-dose treatment with cabergoline induces macroprolactinoma shrinkage. J Clin Endocrinol Metab 1997; 82:3574-79.

16.Mounier C, Trouillas J, Claustrat B, Duthel R, Estour B. Macroprolactinaemia associated with prolactin adenoma. Hum Reprod 2003; 18:853-7.

17.Hauache OM, Rocha AJ, Maia AC, Maciel RM, Vieira JG. Screening for macroprolactinaemia and pituitary imaging studies. Clin Endocrinol (Oxf) 2002; 57:327-31.

18.Al-Mefty O, Kersh JE, Routh A, Smith RR. The longterm side effects of radiation therapy for benign brain tumors in adults. J Neurosurg 1990; 73:502-12.

19.Kuo JS, Chen JC, Yu C, et al. Gamma knife radiosurgery for benign cavernous sinus tumors: quantitative analysis of treatment outcomes. Neurosurgery 2004; 54:1385-93.

20.Kondziolla D, Nathoo N, Flickinger JC, Niranjan A, Maitz AH, Lunsford LD. Long-term results after radiosurgery for benign intracranial tumors. Neurosurgery 2003; 53:815-21.

21.Vladyka V, Lišèák R, Novotný J Jr, Marek J, Jezkova $\mathrm{J}$. Radiation tolerance of functioning pituitary tissue in gamma knife surgery for pituitary adenomas. Neurosurgery 2003; 52:309-16. 